\title{
INJURIES RESULTING FROM PRACTICING PERFORMANCE SPORTS IN TABLE TENNIS AND TENNIS - REVIEW
}

\author{
Mocanu Mircea-Dan ${ }^{1}$, Claudiu Mereuță2 ${ }^{2}$ Daniel-Andrei Iordan ${ }^{2 *}$ \\ 1,2'Dunărea de Jos”' University, Faculty of Physical Education and Sports, 63-65 Gării \\ Street, Galați, România \\ *Corresponding author: Daniel-Andrei Iordan, email: daniel.iordan@ugal.ro
}

\begin{abstract}
Performance sport as an area of human excellence requires, besides an over-average genetic endowment of motor skills, a huge workload of high intensity, which causes the wear of the body systems of the athletes, especially the osteo-musculo-articular. The high ball movement speed for rocket and paddle sports disciplines such as table tennis and field tennis, intensively demands the performance of the body of practitioners when performing specific technical tactics, especially the technical elements of the attack, such as topspin or smash. Based on the theoretical documentation, I noticed an acute condition in the scapulo-humeral joint 21.05\%, at the lumbar level $15.79 \%$ and $13.16 \%$ in the ankle joint in the table tennis and in the field tennis as percentages at scapulo-humeral joint 13.85\%, lumbar $15.38 \%$ and $20.00 \%$ ankle joint, aspects that validate our desire to improve the quality of life of practicing athletes of the two disciplines through a program of amelioration of compensatory nature.

The objective of this theoretical research is to identify the areas subject to mechanical stress with high risk of wear or injury from the two "sister" sports disciplines.
\end{abstract}

Tasks: Critical analysis of a larger volume of studies conducted on the direction of our research interest.

Purpose: obtaining the information necessary which would highlight the risks the performance athletes, practitioners of the two disciplines, are subjected to, as well as gaining knowledge on tennis and table tennis.

Keywords: injuries, tennis, table tennis, kinetotherapy

\section{Introduction}

Today, in sport, high performance requirements have become much more with the participation and increase in training, leading to over-exercise in sports practice resulting inevitably towards a frequency of accidents. An old and confirmed finding in medicine, making it easier to prevent a disease than to treat it, is also perfectly true with regard to performance sports accidents (20). Rocket and pallet sports disciplines are a form of dynamic exercise in which we have many healthy health benefits over the years. Most tennis and table tennis players may suffer asymmetric musculoskeletal changes due to repeated and rapid movements of the trunk in 
flexion and extension in the sagittal and frontal plane where twisting movements are very common in this sports and over time the risk of injury increases .(1,21,4,18).

Table Tennis is a high-quality sport that requires athletes to execute in a short period of time efficient thinking and a strong blow to the ball. Therefore, due to the results of scientific research, table tennis players have a significantly shorter reaction time than any other athlete (2) and even shorter than tennis players $(19,2,3)$.This sport called table tennis is complex, requiring and involvingin the technical sphere different combinations of acceleration and deceleration, changes of direction and balance, in order to be able to hit effectively (16), in the case of tennis, these aspects being similar in a higher stress regime due to the higher weight of the playing materials, to specific stresses that induce great stress on the joints, muscles and spine, which can generate in some cases disorders in the body's mechanisms and systems that are involved in the effort.

Tennis play is characterized by overhead motions, quick starts and stops with short explosive bursts of motion, and a dynamic exchange of intricate strokes and serves, any weakness in the kinetic chain results in dysfunction that creates more reliance on other body segments, leading to injury, athletes are susceptible to a variety of injuries, upper extremity injuries are common and typically from high-velocity arm movements, the shoulder and elbow are most frequently affected followed by the wrist and hand (17).

Also, they compared the muscle activity of the lower limbs during seven typical table tennis strokes, in which they found that the forehand smash and topspin stroke techniques exhibited significantly higher emg amplitudes as compared with other strokes, situation which we consider to be similar in the case of the component technical elements from tennis's specific attack due to the substantial muscle impulse necessary for their execution (6).

The information provided by one of the most recent epidemiological studies on tennis traumas states that upper limb injuries account for $28 \%$ of all injuries for male adult players and $23 \%$ for female adult players, while the shoulder joint was reported to be the most often injured area of the upper limb. Traumas to the shoulder joint are very frequent in professional tennis players, mainly due to the repetitive mechanical overload of the shoulder joint. Shoulder pain was present in $24 \%$ of top tennis players with ages comprised between 12 and 19 years old (9).

Muscle misalignment caused by repetitive unilateral movements (15) that cause stress on fascial tissues, intervertebral discs, spinal ligaments and not only. As a symptomatology, it shows headaches, pain in the cervico-dorso-lumbar region, fatigue but also possible spinal deformities. Trauma in sports seems to increase at all athletic levels due to intense participation, where intensity and requirements are high, and training periods are long (11). Treatment should be very 
effective and should start as soon as possible because most athletes want to return to training and competitions in the shortest possible time (12).

\section{Characteristics}

The biomechanics of the execution of some technical elements common to the two sports is made in three phases or stages, these being the preparation of the hit, the actual hit and the end of the movement, the difference being the amplitude necessary for a high performance execution that we consider to be higher in tennis, as compared to table tennis. Being characterized by explosive motor actions (performed at high speed) involving the entire osteo-musculoskeletal system, the procedures in the sphere of attack involve a greater stress than those of control or middle game (slice, backspin, counter, stop, volleys, drop-shot), reason for which they represent an important factor in the occurrence of specific injuries among others responsible elements, such as a poor recovery after training and competitions, improper dosage of effort during the training period (intensity, volume, complexity), the lack of food and relaxation rigor.

Also, a deficient posture negatively influences the efficiency of some elements specific to the attack according tothe coaches interviewed based on opinion survey, the smash, topspin and retopspin or counter-loop and service being the most important of them, as an example according to Mocanu, Negulescu, Moisescu, (2018), the forehand topspin is considered the most used attack hit in competitive table tennis with an usage percentage of 95.23\%), it is omnipresent both in offensive and defensive players, which highlights both the usage level and also the wearing the athlete's body is submitted to de to their frequent usage.

According to Kondrič, M., et al., (2011) the most often impairments in the racket sports with are:

- $\quad$ Pain in lumbosacral areas;

- $\quad$ Tendinitis of the rotator cuff shoulder's muscles (dead hand);

- $\quad$ King-Kong arm (overdevelopment of the skilled arm);

- $\quad$ Tennis player's elbow (inflammation of the lateral epicondyle);

- $\quad$ Blocking of the forearm nerve;

- $\quad$ Lesion of the abdominal wall.

Two of the most important technical procedures common to the two sports are presented in the images below, in which the muscular, articular and spine effort is particularly high, as it can be seen from the captured details, aspect which we consider responsible as part of the factors meant to create specific disorders, due to the unilateral repetitive nature of the executions. 


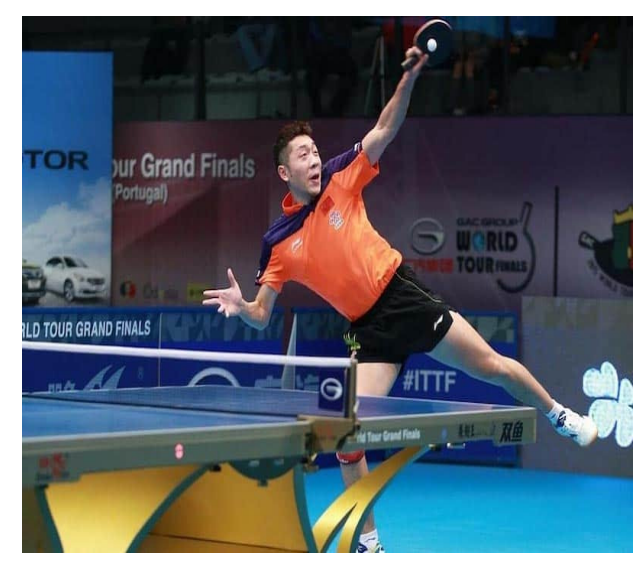

Fig 1. Forehand smash in table tennis- (25)

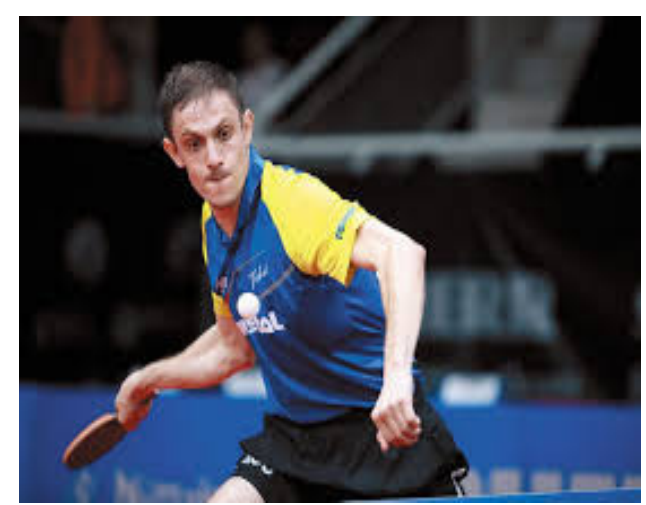

Fig 3. Topspin forehand initial phase-(27)

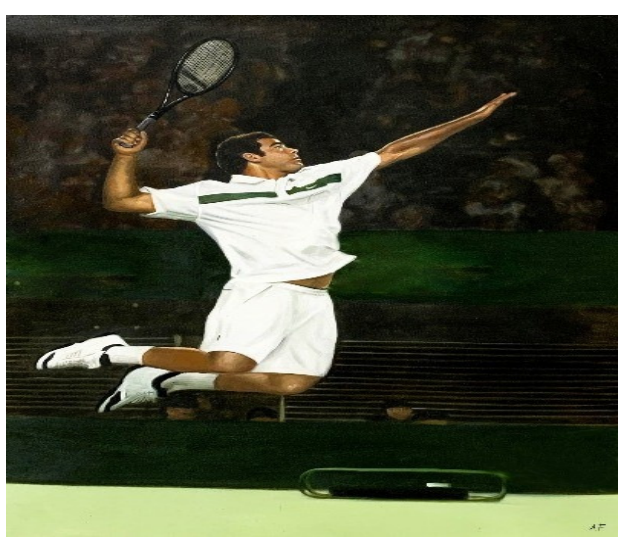

FIG 2. Forehand smash in tennis-(26)

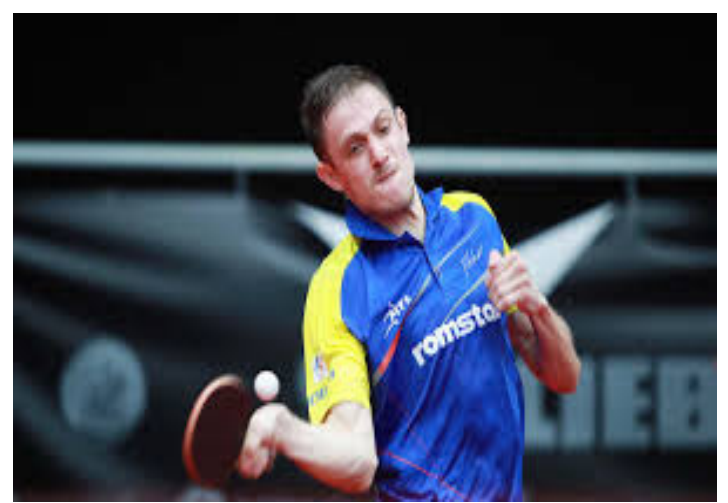

Fig 4. Topspin forehand hitting phase-(28)

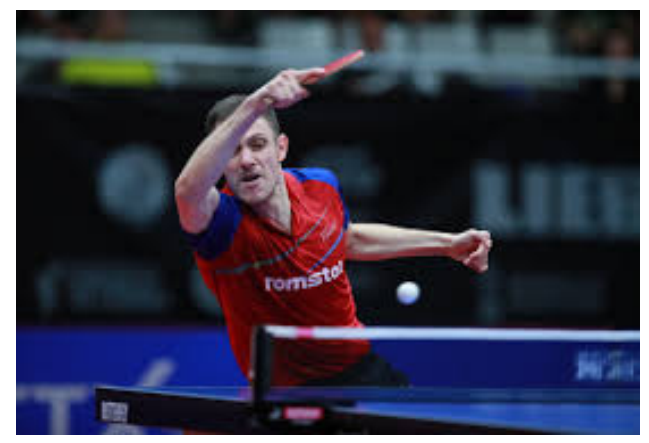

Fig. 5. Topspin forehand finish-(29) 


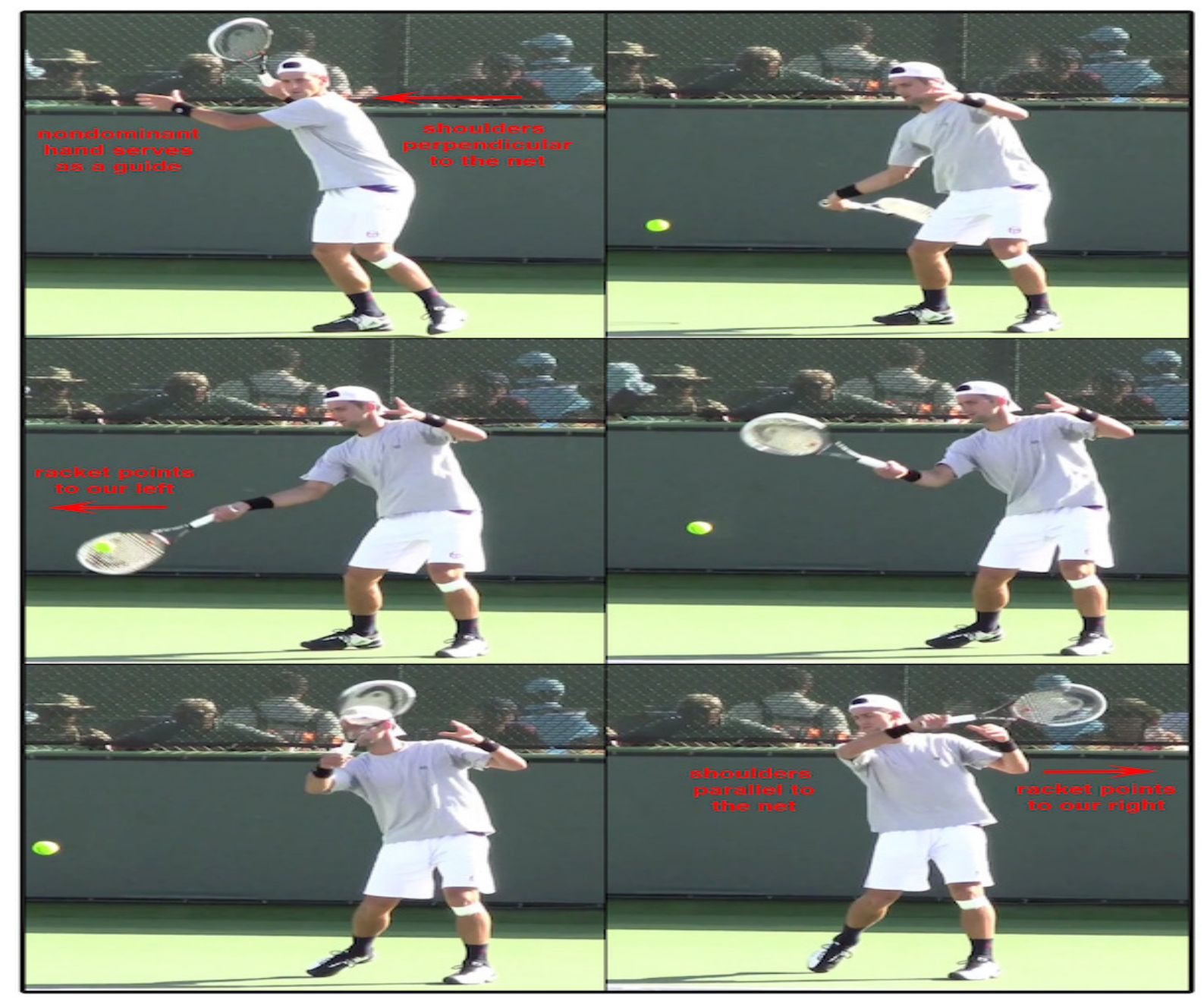

Fig. 6. Topspin forhand in tennis frame by frame-(30)

Instead of doing trunk rotates(FIG.7), we make this twist of the lumbar spine(FIG.8), when we speak about twist, this is composed of 3 movements: - flexion, inclination and rotation, this is the basic mechanism of the hernia disc.

In this two sports, asymmetric changes due to the single-arm stroke.

And yes, sports activity, performance sports can cause lumbar pain, why, because all the vertebral plate is distorted. As physiotherapists, promoters of kinetotherapy and healthy life, we need to help and teach people, athletes and performance athletes, how to include new methods and techniques of relaxation, stretching, and, most importantly, the reposturing part, the balance part of the muscular system and respectively the bone system, because the muscles are the cranes that draw bones in all parts. 


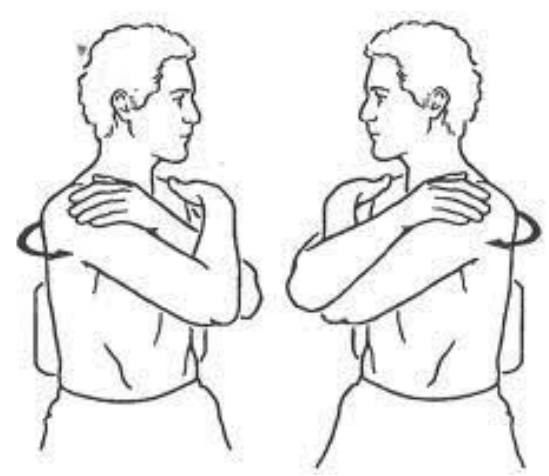

Fig.7 rotation trunk-(17)

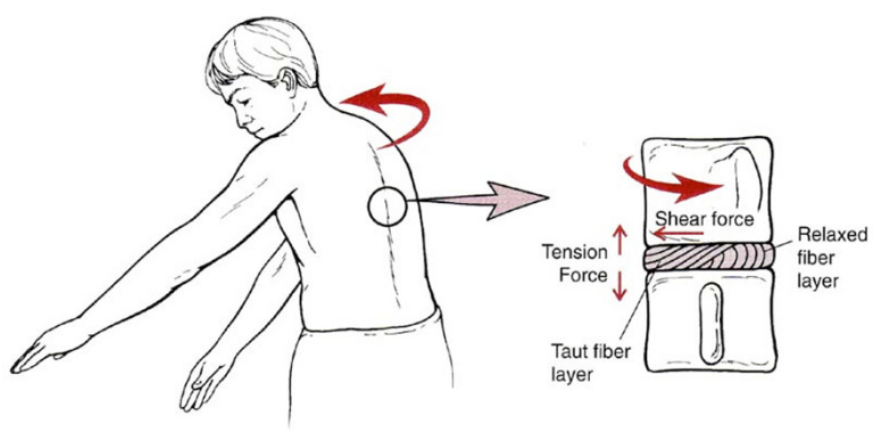

Fig. 8 twisttrunk

Source: Hamill and KnutzenK, 1995, 'Biomechanical Basis of Human Movement,' Lippincott

\section{Methods}

In order to carry out this scientific research on the specific injuries of the two related disciplines, we mainly used the bibliographic study method, this being responsible for most of the information found in the presented material.

The logical and the hermeneutic method facilitated the search, the choice and interpretation of the selected passages regarding the information of interest, the method of registration based on survey also playing an important role in obtaining and highlighting some data that we consider valuable for designing the present scientific article.

Also, the statistical-mathematical method was a test indicator for interpreting the data collected from the interviewed specialists, the used program being SPSS vers. 23.

\section{Results}

It is also important to mention that in a study based on an opinion survey in table tennis conducted by Iordan, Mocanu\&Mereuta (2020) on the 5 areas of the spine subjected to high wear and implicitly to the appearance of disorders,the area presenting the most frequent incidence rate was indicated to be the lumbar area with a percentage of $78 \%$.

The biomechanical characteristics of these two sports are:

(a) asymmetric changes (resulting from strong, repetitive single-arm strokes);

(b) strong lumbar and hip joint rotation. (21)

In this review, 68 questionnaires were completed by top Slovenian athletes. Of which 29 are table tennis players and 39 tennis players.

The average age of questioned athletes was 19.37 years. (9) 


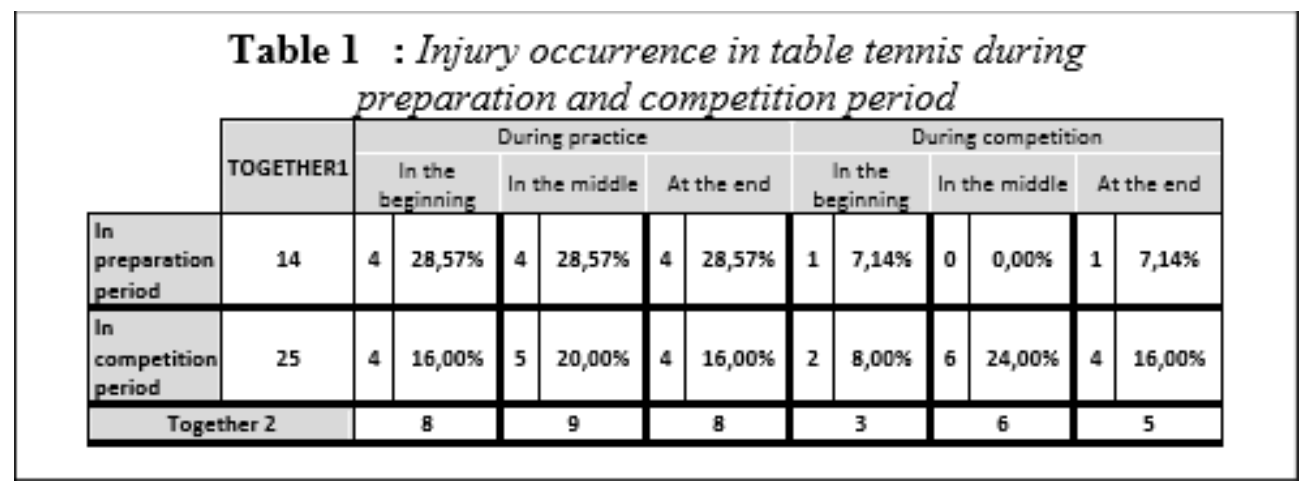

Table 1, 1.2, 1.3 acording to Kondrič, M., Matković, B., Furjan-Mandić, G., Hadžić, V., \&Dervišević, E. (2011). Injuries in racket sports among Slovenian players. Collegium antropologicum, 35(2), 413-417.

Table 1.2: Injury location

\begin{tabular}{|l|r|r|}
\hline & Table tennis & \multicolumn{1}{l|}{ Tennis } \\
\hline Head & $0,00 \%$ & $0,00 \%$ \\
\hline Neck & $0,00 \%$ & $4,62 \%$ \\
\hline Shoulder & $21,05 \%$ & $13,85 \%$ \\
\hline Upper arm & $0,00 \%$ & $0,00 \%$ \\
\hline Forearm & $0,00 \%$ & $1,54 \%$ \\
\hline Wrist & $10,53 \%$ & $13,85 \%$ \\
\hline Fingers & $0,00 \%$ & $0,00 \%$ \\
\hline Trunk & $2,63 \%$ & $3,08 \%$ \\
\hline Spine & $15,79 \%$ & $15,38 \%$ \\
\hline Hip & $15,79 \%$ & $1,54 \%$ \\
\hline Femur & $7,89 \%$ & $12,31 \%$ \\
\hline Knee & $2,63 \%$ & $1,54 \%$ \\
\hline Shank & $0,00 \%$ & $3,08 \%$ \\
\hline Ankle & $13,16 \%$ & $20,00 \%$ \\
\hline Foot & $10,53 \%$ & $9,23 \%$ \\
\hline
\end{tabular}

Table 1.3: Percentage of injuries per individual

\begin{tabular}{|l|r|r|r|r|r|}
\hline & Skin & Muscles & Tendon & Bones & Joint \\
\hline $\begin{array}{l}\text { Table } \\
\text { tennis }\end{array}$ & $0,00 \%$ & $52,63 \%$ & $13,16 \%$ & $13,16 \%$ & $21,05 \%$ \\
\hline Tennis & $0,00 \%$ & $49,23 \%$ & $10,77 \%$ & $4,62 \%$ & $35,38 \%$ \\
\hline
\end{tabular}


(24). During the competition period, injuries are not lacking, as the number of factors is steadily increasing (Table 1). When talking about injuries, most of them take place in the middle of the training session or during the competition. At the same time this is very interesting because it can show us inappropriate heating, the lack of stretching exercises before the game, even after, manipulations but also other causes.

The most common injuries in table tennis and tennis are the muscles tissues (Table 1.3), followed by injuries in the joints and tendons.

As a percentage, injuries to the joint of the shoulder and ankle joint are most common, which also confirms the epidemiology reports worldwide. The shoulder joint is the most flexible part of the body but at the same time it is most prone to injuries. In the two sports, the upper part, the kicks made by the players involve a lot the upper part, the trunk. Amplitude of movement and control of the muscular system at the trunk if it is reduced due to a certain factor that the efficiency and effectiveness of the strokes decreases leading to muscle imbalances resulting in a possible injury.

At present, it is difficult to estimate exact values for the nature of the pains and types of injuries in the lumbar region for table tennis and tennis players due to limited sports-specific research. Surprisingly, in table tennis, due to these sudden movements, the percentage of injuries in the hip joint rises to $15.79 \%$ and it is low for field tennis players where it is (only $1.54 \%$ ), which indicates that most of the Slovenian players play on "soft" ground. Column vertebral injuries (over $15 \%$ ) relate more or less to pain or injuries caused by overload in the lower spine.

\section{CONCLUSIONS}

As a result of the analysis of the results and information taken and presented in the present material, we want to outline some of the most important and interesting aspects of tennis and table tennis on the areas most affected by technical procedures and specific motor skills, so as to create an image clear to the specialists from the two sports disciplines, but also to the enthusiasts who practice the two sports regularly, in order to inform them about the risks to which they or the athletes they train may be subjected so as to be able to prevent such specific pathologies.

While in tennis the areas with a higher percentage incidence of injuries as compared to table tennis are represented by the wrist, femur, ankle and neck muscles, in the sister discipline the shoulder, spine, hip and sole are the most important aspects which highlights the fact that despite the fact that they are racket sports, the game materials and the amplitude of the movements along with the play spaces that must be covered by the movement of athletes create pathologies in different areas of their body,the tendons and bones having a higher percentage of injuries, while in tennis the joints are most prone to create problems for practicing athletes. 
Kinetoteraphy and everything that comes with it is an excellent way to improve skills and all the movements in both sports. Effective management of table tennis and tennis injuries is therefore prevention, based on an understanding of the factors involved in overweight injuries. Frequent repetition of the activity required to develop and improve table tennis and tennis skills can result in chronic injury caused by overloading. Maybe poor technique, muscle training, and inappropriate equipment lead to execution errors that may cause increased stress on muscularmuscular tissues and cause pain due to micro-trauma or overload. In order to minimize the painful response, the body adopts compensatory mechanisms, which eventually add to the execution errors and thus establishes the endless cycle of overloading. Database records contribute to the planning and organization of medical care for performance athletes, which inevitably require better medical supervision and higher quality;

From this perspective, it is therefore very important for a table tennis or tennis coach to work in close collaboration and under the supervision of the physical trainer, team physician and physiotherapist. This review of the literature could be of some use to all technicians involved in table tennis.

A specific postural program and the implementation of orthopedic heel cushions could be recommended to improve the postures, compensatory work through carefully directed actuation systems for a balanced development of the body muscles opposite to the active execution part, as well as the systematic evaluation regarding the bone and muscular composition, the possible existence of inflammations, the posture and the plantar surface in static and dynamic, represent actions meant to prevent the appearance or development of specific pathologies of the osteomuscular-articular system and of the spine.

ACKNOWLEDGMENTS: The present work was supported by ANTREPRENORDOC project, in the framework of Human Researches Development Operational Programme 2014-2020, financed from the European Social Fund under the contract number 36355/23.05.2019 -SIMS code: 123847.

\section{Bibliography}

1. Abrams, G. D., Renstrom, P. A., \& Safran, M. R. (2012). Epidemiology of musculoskeletal injury in the tennis player. Br J Sports Med, 46(7), 492-498.

2. Bhabhor, M. K., Vidja, K., Bhanderi, P., Dodhia, S., Kath-rotia, R., \& Joshi, V. (2013). A comparative study of visual reaction time in table tennis players and healthy controls. Indian Journal of Physiology and Pharmacology, 57, 439-442. 
3. Can, S., Kilit, B., Arslan, E., \&Suveren, S. (2014). The comparison of reaction time of male tennis players, table tennis players and the ones who don't exercise at all in 10 to 12 age group. Nigde University Journal of Physical Educa- tion and Sport Sciences, 8, 195-201.

4. Ellenbecker, T.S., Pluim, B., Vivier, S. and Sniteman, C. (2009) Com- mon injuries in tennis players: Exercises to address muscular imbalances and reduce injury risk. Strength and Conditioning Journal 31, 50-58

5. Hamill, J, \&Knutzen, K, 1995, 'Biomechanical Basis of Human Movement,' Lippincott

6. He, Y., Lv, X., Zhou, Z., Sun, D., Baker, J. S., \& Gu, Y. (2020). Comparing the Kinematic Characteristics of the Lower Limbs in Table Tennis: Differences between Diagonal and Straight Shots Using the Forehand Loop. Journal of Sports Science \& Medicine, 19(3), 522.

7. Iordan Daniel-Andrei, MocanuMircea-Dan, MereutaClaudiu. Topspin's influence on the spine in female juniors III in table tennis. 2020

8. Jayanthi, N., \&Esser, S. (2013). Racket sports. Current sports medicine reports, 12(5), 329-336.

9. Kekelekis, A, Nikolaidis, PT, Moore, IS, Rosemann, T, Knechtle, Risk Factors for Upper Limb Injury in Tennis Players: A Systematic Review INTERNATIONAL JOURNAL OF ENVIRONMENTAL RESEARCH AND PUBLIC HEALTH Volume:17 Issue:8 Article Number:2744 DOI: 10.3390/ijerph17082744

10. Kondrič, M., \&Furjan-Mandić, G. (2003). Zakonitostikondicijskihprogramov $\quad v$ treningunamiznoteniškegaigralca. [Rules for designing physical preparation in table tennis]. Top spin (Ljubl.), $2(5): 3-6$

11. Kondrič, M., Furjan-Mandić, G., Hadžić, V., Dervišević, E., Matković, B., \&Ochiana, N. (2008). Injuries in Slovenian table tennis players compared with injuries of some of the best Slovenian tennis players. Gymnasium. Revista de educatiefizicasi sport, 8(13), 73-85.

12. Kondrič, M., Matković, B., Furjan-Mandić, G., Hadžić, V., \&Dervišević, E. (2011). Injuries in racket sports among Slovenian players. Collegium antropologicum, 35(2), 413-417.

13. Krivickas, L.S. (1997). Anatomical factors associated with overuse sports injuries. Sports Medicine. 24(2):132-146.

14. Mocanu, m., Negulescu, i. c. \&Moisescu, c.,p., (2018). Optimization of the topspin performance biomechanics in female juniors in table tennis. Discobolul, 50. https://dx.doi.org/10.15405/epsbs.2019.02.4

15. Muyor, J. M., Sánchez-Sánchez, E., Sanz-Rivas, D., \&López-Miñarro, P. A. (2013). Sagittal spinal morphology in highly trained adolescent tennis players. Journal of sports science \& medicine, 12(3), 588.

16. Nikolakakis, A., Mavridis, G., Gourgoulis, V., Pilianidis, T., \&Rokka, S. (2020). Effect of an intervention program that uses elastic bands on the improvement of the forehand topspin stroke in young table tennis athletes. Journal of Physical Education and Sport, 20, 2189-2195.

17. Patel, H., Lala, S., Helfner, B. et al. Tennis overuse injuries in the upper extremity. Skeletal Radiol (2020). https://doi.org/10.1007/s00256-020-03634-2

18. Pluim, B.M., Staal, J.B., Windler, G.E. and Jayanthi, N. (2006) Tennis injuries: occurrence, aetiology, and prevention. British Journal of Sports Medicine 40, 415-423.

19. Pradas, F., Carrasco, L., Martinez, E., \& Herrero, R. (2007). Anthropometric profile, somatotype, and body composition of young table tennis players. RevistaInternacional de Ciencias del Deporte, 3(7), 11-23.

20. Scott, M.J. (1992). ITTF questionare of elite athletes at 41st World table tennis championships. International Journal of Table Tennis Sciences, No. 1, pp. 191-193 
21. Weihua S., Yulan Z.(2014). Present situation investigation and prevention strategy analysis of the college table tennis players' sports injury. BTAIJ, 10(9), 2014 [3704-3710]

22. http://tenisdemasa-valeriu.blogspot.com/2012/03/lovituri-liftate-topspin.html

23. http://www.sport-inromania.ro/articole/tenis-de-masa-ovidiu-ionescu-argint-european/

24. https://www.poole.nhs.uk/pdf/Thoracic\%20and\%20Lumbar\%20Spine\%20ROM\%20DB\%20ver2\%2020.9.1

7.pdf

25. http://www.range-of-motion-table-tennis.jpg $(800 \times 533)$ (pingpongruler.com)

26. http://www.7be843_724baf99648140958364c4ce8bb836bb mv2.jpg (400×533) (wixstatic.com)

27. http://www. $1200 \times 800($ google.com $)$

28. http://www. IONESCU-Ovidiu-ROU_2018EC_PRG_6855.jpg (1920×1080) (jurnalistii.ro)

29. http://www. $5472 \times 3648$

30. http://www djoforehand_600.png (600×933)(oliverelliott.org) 